\section{LA-UR-08- 6061}

Approved for public release;

distribution is unlimited.
Title: Localized Ferromagnetic Resonance Force Microscopy of Permalloy-Cobalt Films

Author(s): $\quad$ E. Nazaretski, R. Movshovich, I. Martin, Y. Obukhov, D.V. Pelekhov, K.C. Cha, E.A. Akhadov, P.C. Hammel

Applied Phys. Ltrs.

Los Alamos National Laboratory, an affirmative action/equal opportunity employer, is operated by the Los Alamos National Security, LLC for the National Nuclear Security Administration of the U.S. Department of Energy under contract DE-AC52-06NA25396. By acceptance of this article, the publisher recognizes that the U.S. Government retains a nonexclusive, royalty-free license to publish or reproduce the published form of this contribution, or to allow others to do so, for U.S. Government purposes. Los Alamos National Laboratory requests that the publisher identify this article as work performed under the auspices of the U.S. Department of Energy. Los Alamos National Laboratory strongly supports academic freedom and a researcher's right to publish; as an institution, however, the Laboratory does not endorse the viewpoint of a publication or guarantee its technical correctness. 


\title{
Localized ferromagnetic resonance force microscopy of permalloy-cobalt films
}

\author{
E. Nazaretski, ${ }^{1}$ Yu. Obukhov, ${ }^{2}$ I. Martin, ${ }^{1}$ D. V. Pelekhov, ${ }^{2}$ \\ K. C. Cha, ${ }^{1}$ E. A. Akhadov, ${ }^{1}$ P. C. Hammel, ${ }^{2}$ and R. Movshovich ${ }^{1}$ \\ ${ }^{1}$ Los Alamos National Laboratory, Los Alamos, NM 87545 \\ ${ }^{2}$ Department of Physics, Ohio State University, Columbus OH 43210,
}

\begin{abstract}
We report the Ferromagnetic Resonance Force Microscopy (FMRFM) experiments on a combined permalloy-cobalt continuous film. Our studies demonstrate the capability of FMRFM to perform local spectroscopy of different ferromagnetic materials. Theoretical analysis of the uniform resonance mode at the edge of the film provides good quantitative agreement with the experimental data. Our experiments demonstrate the micron scale lateral resolution and allow to extract local magnetic properties in continuous ferromagnetic samples.
\end{abstract}

Magnetic resonance force microscopy (MRFM) attracted a lot of interest in the past decade due to its high spin sensitivity and excellent spatial resolution in paramagnetic and nuclear spin systems.[1-5] MRFM studies on ferromagnetic samples have been also performed. [69] Contrary to the paramagnetic and nuclear magnetic resonance imaging experiments, $[10,11]$ ferromagnetic resonance (FMR) poses a challenge for spatially resolved studies due to the presence of a strong exchange coupling between the spins. Observed resonance spectra usually involve precession of the entire magnetization in the sample and locally resolved FMR is not possible with the conventional resonance techniques. For technological applications it is important to develop the experimental approach capable of localized imaging of magnetic properties in continuous ferromagnetic media. In this Letter, we report the FMRFM experiments performed on a combined permalloy (Py) - cobalt (Co) continuous film and demonstrate the capability of FMRFM to perform local spectroscopy of ferromagnetic materials.

The permalloy-cobalt sample is schematically shown in Fig. 1. A $20 \mathrm{~nm}$ thick Ti film was uniformly applied onto the surface of a $100 \mu \mathrm{m}$ thick Si (100) wafer. 20 $\mathrm{nm}$ of Co was deposited into a rectangular area $(2.5 \times$ $5 \mathrm{~mm}$ ) defined in photoresist followed by the lift-off. A complimentary rectangular area of $20 \mathrm{~nm}$ thick Py was similarly defined and deposited. The entire structure was then coated with a $20 \mathrm{~nm}$ thick layer of Ti. The interface between $\mathrm{Co}$ and Py regions was examined in a scanning

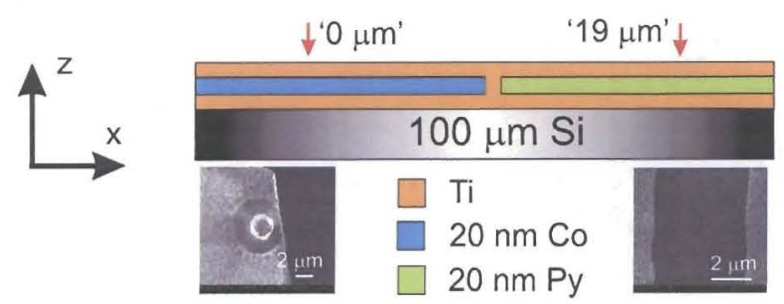

FIG. 1: Schematic of the Co - Py sample. The arrows mark the scan range for spectra shown in Fig. 2. The SEM image on the right shows the gap between Py and Co. The SEM image on the left depicts the cantilever tip. electron microscope (SEM) and revealed the gap with the width varying between 3 and $6 \mu \mathrm{m}$ along the entire length of the sample (see SEM image in Fig. 1). An approximately $1.7 \times 1.7 \mathrm{~mm}^{2}$ piece was cut and glued to the strip line resonator of the FMRFM apparatus and the film plane was oriented perpendicular to the direction of the external magnetic field $H_{\text {ext }}$. For FMRFM studies we used the cantilever with the spherical magnetic tip (see SEM image in Fig. 1) and its spatial field profile has been carefully characterized [12]. More details on the experimental apparatus can be found in Ref. [13]

In Fig. 2 we show the evolution of the FMRFM signal as a function of the lateral position and the applied magnetic field. The cantilever was scanned across the interface between $\mathrm{Co}$ and $\mathrm{Py}$, in the region schematically marked with arrows in Fig. 1. The FMRFM signal was recorded in two different regions of $H_{\text {ext }}$ which correspond to Py and Co resonance fields for the microwave frequency of $f_{R F}=9.35 \mathrm{GHz}$. Insets in Fig. 2 show the evolution of the FMRFM spectra as a function of the lateral position. The structure of the signal is reminiscent of those reported earlier in Ref. [14] and is comprised of two distinctive contributions. The first one, is the negative signal which occurs at lower values of $H_{e x t}$ and originates from the region of the sample right under the cantilever tip where the probe field is strong and positive (localized resonance). The second contribution is positive and is observed at higher values of $H_{e x t}$. This part of the signal corresponds to the larger region of the sample where the tip field is weak and negative (uniform contribution). As seen in Fig. 2, at the beginning of the lateral scan the negative (lower field) resonance structure is presented only in the Co spectra (see inset 'Co $3 \mu \mathrm{m}$ '). At around $9 \mu \mathrm{m}$ of the lateral position there is no local contributions neither in Py nor in Co resonances (region above the gap between Py and Co films). As we continued scanning into the Py part of the sample the negative (local) resonance structure is observed in the Py resonance (inset 'Py $19 \mu \mathrm{m}$ '), while the Co spectrum remains unchanged (inset 'Co $19 \mu \mathrm{m}$ ').

It is convenient to start the analysis of the FMRFM signals considering the uniform resonance in the film e.g. 


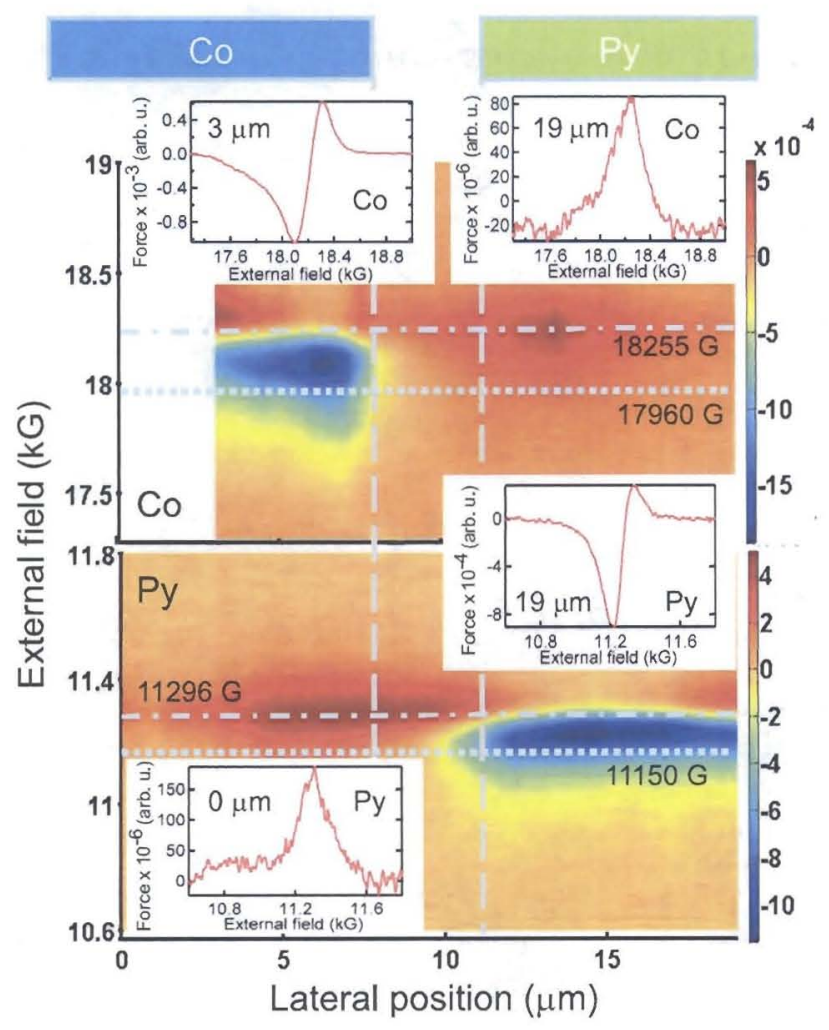

FIG. 2: FMRFM force image as a function of $H_{\text {ext }}$ and the lateral position. We show the Co and Py forces in the upper and lower panels respectively. Insets demonstrate the evolution of the FMRFM signal as a function of the lateral position. Vertical dashed lines show the boundaries of the Co and Py films. The horizontal dashed-doted lines are drawn through the values of $\mathrm{H}_{\text {ext }}=18255 \mathrm{G}$ for $\mathrm{Co}$ and $\mathrm{H}_{\text {ext }}=11296 \mathrm{G}$ for Py respectively and correspond to the uniform resonance (see Fig. 3b). The horizontal dotted lines at $\mathrm{H}_{\text {ext }}=17960 \mathrm{G}$ and $\mathrm{H}_{\text {ext }}=11150 \mathrm{G}$ for $\mathrm{Co}$ and Py respectively, demonstrate the spatial resolution of the FMRFM (see Fig. 3c). Experimental parameters: $\mathrm{T}=11 \mathrm{~K}, f_{R F}=9.35 \mathrm{GHz}$, probe-sample distance $\approx 5.6 \mu \mathrm{m}$.

the case when the entire dynamic magnetization $m$ is constant and the resonance field is only weakly affected by the probe. This case is valid for the large probe-sample distances (insets 'Co $19 \mu \mathrm{m}$ ', and 'Py $0 \mu \mathrm{m}$ ' in Fig. 2). The frequency of the uniform resonance in a thin film can be written as $\omega_{R F} / \gamma=H_{e x t}-4 \pi M_{s}$, where $4 \pi M_{s}$ is the saturation magnetization and $\gamma$ is the gyromagnetic ratio. FMRFM spectra shown in 'Co $19 \mu \mathrm{m}$ ' and 'Py $0 \mu \mathrm{m}$ ' insets in Fig. 2 yield the values of $4 \pi M_{s}=8052 \mathrm{G}$ for Py and $4 \pi M_{s}=15013 \mathrm{G}$ for Co respectively. Obtained values of $4 \pi M_{s}$ are used for the Magnetic Force Microscopy (MFM) calibration of the probe-sample distance. During the dynamic MFM measurements the cantilever was scanned across the Py - Co interface and changes in its resonance frequency were recorded. The gradient of the MFM force for a half infinite film can be written as fol-

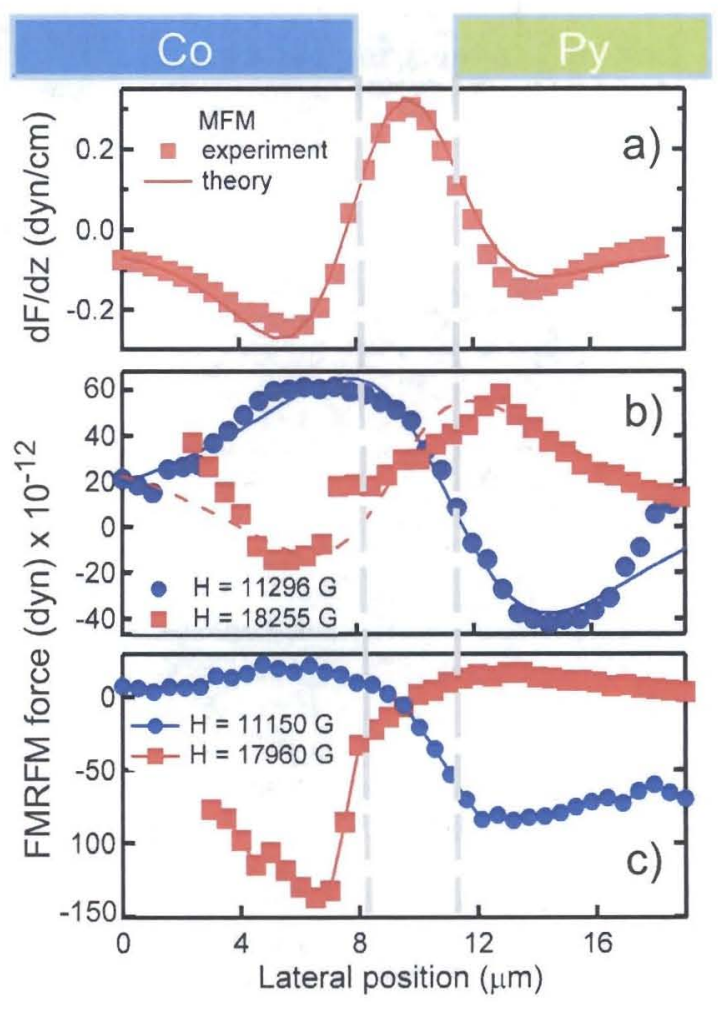

FIG. 3: a) MFM data acquired at $\mathrm{H}_{\text {ext }}=18255 \mathrm{G}$, solid line is the fit to Eq. 1. b) FMRFM force data for the uniform FMR modes. $\mathrm{H}_{\text {ext }}=18255 \mathrm{G}$ for $\mathrm{Co}$ (squares) and $\mathrm{H}_{\text {ext }}=$ $11296 \mathrm{G}$ for Py (circles). Solid and dashed lines are the fits using Eq. 2. Fit parameters: $m / M_{s}=0.0014, \beta=0.65$ for Co and $m / M_{s}=0.0028, \beta=0.5$ for Py. c) FMRFM force for the localized (close to the probe) FMR mode acquired at $\mathrm{H}_{e x t}=17960 \mathrm{G}$ for Co (squares) and $\mathrm{H}_{e x t}=11150 \mathrm{G}$ for $\mathrm{Py}$ (circles). The lateral resolution is better than $2 \mu \mathrm{m}$.

lows:

$$
\frac{\partial F}{\partial z}=4 m_{p} M_{s} L \frac{x\left(x^{2}-3 z^{2}\right)}{\left(x^{2}+z^{2}\right)^{3}}
$$

where $m_{p}=7 \times 10^{-9} \mathrm{emu}$ is the probe magnetic moment [12] and $L$ is the film thickness. $z$ is the probe-film distance and $x$ is the lateral position with respect to the film edge $(x \geq 0)$. MFM data were acquired at $\mathrm{H}_{\text {ext }}=$ $18255 \mathrm{G}$ thus both films were saturated. The MFM data and the fit to Eq. 1 are shown in Fig. 3a, yielding the tip-sample distance of $z \approx 5.6 \mu \mathrm{m}$ (here and later the tip-sample distance includes the probe radius of $\approx 1.2$ $\mu \mathrm{m})$ and the films boundaries $(x \leq 8 \mu \mathrm{m}$ for Co and $x \geq$ $11 \mu \mathrm{m}$ for Py).

The tip field suppresses the uniform FMR mode from the region under the tip, and according to Obukhov et al. [15] the magnitude of the suppression depends on the probe-sample distance. It is described as partial suppression at distances $z \gg \sqrt{\frac{2 m_{p}}{\pi M_{s} L \alpha_{0}}}\left(\alpha_{0}\right.$ is the first 
zero of the Bessel function $J_{0}\left(\alpha_{0}\right)=0$ ) and full suppression at $z \ll \sqrt{\frac{2 m_{p}}{\pi M_{s} L \alpha_{0}}}$. The spatial confinement of the suppression is defined as $r=\sqrt{2} z$. FMRFM data discussed in this Letter are at the boundary of these two regions, thus it is legitimate to consider the regime of full suppression, however introduce the magnitude of the suppression as a fit parameter. Detected FMRFM force originates from the variation of the $z$-component of the film magnetization caused by the amplitude modulation of the dynamic magnetization $m$ and can be written as $F=M_{z} L \cdot \partial H_{p} / \partial z=\sqrt{M_{s}^{2}-m^{2}} L \cdot \partial H_{p} / \partial z \approx$ $-m^{2} L / 2 M_{s} \cdot \partial H_{p} / \partial z$, where $H_{p}$ is the probe field. The total FMRFM force in our approximation can be then written as follows:

$F=-\frac{m^{2}}{2 M_{s}} L\left(\frac{4 x z m_{p}}{\left(x^{2}+z^{2}\right)^{2}}-\beta \int_{S} \theta\left(x^{\prime}\right) \frac{\partial H_{p}}{\partial z}\left(x-x^{\prime}\right) d r^{\prime}\right)$,

where the first term describes the force between the probe and the half infinite film and the second term pertains to the force between the probe and the area of radius $r=\sqrt{2} z$ under the tip. The Heaviside function $\theta\left(x^{\prime}\right)$ represents the fact that the film is positioned at $x^{\prime} \geq 0$ and the dimensionless parameter $\beta$ quantifies the magnitude of the suppression of the uniform FMR mode. In Fig. $3 b$ we plot the experimental data extracted from Fig. 2 and corresponding fits using Eq. 2. Fig. 3b demonstrates reasonable qualitative and quantitative agreement between theory and experiment and proofs the validity of the model. It is important to mention that in our model we assume the dynamic magnetization $m$ to be constant throughout the film. However, $m$ may vary due to the change of the demagnetizing field e.g. $-4 \pi M_{s}$ far from the film boundary and $-2 \pi M_{s}$ at the film boundary. Our estimates show that $m$ changes from a constant value in the film down to zero at the film edge. The length scale of this change equals to $\pi M_{s} L / \Delta H(\Delta H$ is the linewidth of the uniform resonance) and yields the value of $\approx 1 \mu \mathrm{m}$, small compare to the probe-sample distance thus only weakly affecting the fits shown in Fig. 3b.

The spatial resolution of the uniform FMR mode shown in Fig. $3 \mathrm{~b}$ is comparable to the MFM lateral resolution depicted in Fig. 3a and is determined by the probe sample distance of $\approx 5.6 \mu \mathrm{m}$. However, it can be further improved by tracking the intensity of the FMRFM signal at values of $\mathrm{H}_{e x t}$ lower than that of the uniform FMR mode (insets 'Co $3 \mu \mathrm{m}$ ' and 'Py $19 \mu \mathrm{m}$ ' in Fig. 2). In Fig. $3 \mathrm{c}$ we show the FMRFM force acquired at $\mathrm{H}_{\text {ext }}=$ $17960 \mathrm{G}$ for $\mathrm{Co}$ and $\mathrm{H}_{\text {ext }}=11150 \mathrm{G}$ for Py respectively (values of $\mathrm{H}_{\text {ext }}$ are schematically indicated with dotted lines in Fig. 2). The contribution to FMRFM signal at lower values of $\mathrm{H}_{\text {ext }}$ originates from the highly localized region of the sample under the probe. As seen in Fig. 3c the lateral resolution is better than $2 \mu \mathrm{m}$ and is determined by the FMR resonance linewidth and the spatial profile of the FMR mode under the tip. Further theoret- ical and numerical analysis is required to understand the evolution of the FMR modes excited under the probe in the presence of a strongly inhomogeneous tip field and boundaries of the sample.

In conclusion, we have demonstrated the capability of FMRFM to perform local spectroscopy of different ferromagnetic materials. Proposed model for the uniform FMR mode provides good agreement between theory and experiment. The demonstrated spatial resolution for the tip induced resonance is better than $2 \mu \mathrm{m}$.

The work performed at Los Alamos was supported by the US Department of Energy, and Center for Integrated Nanotechnologies at Los Alamos and Sandia National Laboratories. The work at OSU was supported by the US Department of Energy through grant DE-FG0203ER46054.

[1] D. Rugar, C. S. Yannoni, and J. A. Sidles, Nature 360, 563 (1993)

[2] D. Rugar and O. Züger and S. Hoen and C. S. Yannoni and H. M. Vieth and R. D. Kendrick, Science 264, 1560 (1994)

[3] Z. Zhang, M. L. Roukes, and P. C. Hammel, J. Appl. Phys. 80, 6931 (1996)

[4] D. Rugar, R. Budakian, H. J. Mamin, and W. Chui, Nature 430, 329 (2004)

[5] C. L. Degen, Q. Lin, A. Hunkeler, U. Meier, M. Tomaselli, and B. H. Meier, Phys. Rev. Lett. 94, 207601 (2005)

[6] Z. Zhang, P. C. Hammel, and P. E. Wigen, Appl. Phys. Lett. 68, 2005 (1996)

[7] G. de Loubens, V.V. Naletov, O. Klein, J. Ben Youssef, F. Boust, and N. Vukadinovic, Phys. Rev. Lett. 98, 127601 (2007)

[8] Yu. Obukhov, D. V. Pelekhov, J. Kim, P. Banerjee, I. Martin, E. Nazaretski, R. Movshovich, S. An, T. J. Gramila, S. Batra, P. C. Hammel, Phys. Rev. Lett. 100, 197601, (2008)

[9] E. Nazaretski, J. D. Thompson, M. Zalalutdinov, J. W. Baldwin, B. Houston, T. Mewes, D. V. Pelekhov, P. Wigen, P. C. Hammel, and R. Movshovich, J. Appl. Phys. 101, 074905 (2007)

[10] O. Züger, and D. Rugar, Appl. Phys. Lett. 63, 2496 (1993)

[11] H.J. Mamin, M. Poggio, C. L. Degen, D. Rugar, Nature Nanotech. 2, 301 (2007).

[12] E. Nazaretski, E. A. Akhadov, I. Martin, D. V. Pelekhov, P. C. Hammel, and R. Movshovich, Appl. Phys. Lett. 92, 214104 (2008).

[13] E. Nazaretski, T. Mewes, D. Pelekhov, P. C. Hammel, and R. Movshovich, AIP Conf. Proc. 850, 1641 (2006)

[14] E. Nazaretski, D. V. Pelekhov, I. Martin, M. Zalalutdinov, J. W. Baldwin, T. Mewes, B. Houston, P. C. Hammel, and R. Movshovich, Appl. Phys. Lett. 90234105 (2007)

[15] Yu. Obukhov, D. V. Pelekhov, E. Nazaretski, R. Movshovich, P. C. Hammel, submitted to Appl. Phys. Lett. (2008) 